\title{
ICDD full diffraction pattern polymer project for biomedical materials characterization
}

Thomas Nelson Blanton ${ }^{1}$, Stacy Gates-Rector ${ }^{1}$, Timothy Fawcett $^{1}$, Scott Misture $^{2}$

${ }^{1}$ Icdd, Newtown Square, United States, ${ }^{2}$ Alfred University, Alfred, United States E-mail: tblanton@icdd.com

Polymers show a range of order from amorphous to semi-crystalline. Traditional organic analytical techniques, such as infrared spectroscopy (IR), differential scanning calorimetry (DSC), thermal gravimetric analysis (TGA), and nuclear magnetic resonance (NMR), are typically used for polymer characterization. Though X-ray diffraction (XRD) is not commonly used as the primary technique for polymer characterization, XRD does provide unique information about a polymer particularly when assessing crystallinity and crystallite size. In medical applications, polymers are often used as excipients in pharmaceuticals, and the base material for delivery devices used in biomedical applications.

ICDD has been adding polymer diffraction data to the Powder Diffraction File ${ }^{T M}$ (PDFß) with the focus on adding raw data diffraction patterns (1D and 2D) as part of the PDF entry. The inclusion of the raw data diffraction pattern is important in correctly identifying the polymer contribution to a composite material diffraction pattern. A traditional d-spacing/intensity stick pattern or simulated diffraction pattern is not capable of accounting for the full-pattern diffraction profile of polymers since all polymers have some amorphous component. The ICDD polymer project focuses on industrially important polymers with an added emphasis on polymers used in medical and biomedical applications. New entries resulting from this project will be presented along with an interesting finding for a substituted cellulosic that crystallizes when heated; however, the crystalline phase is not consistent with the parent cellulosic.

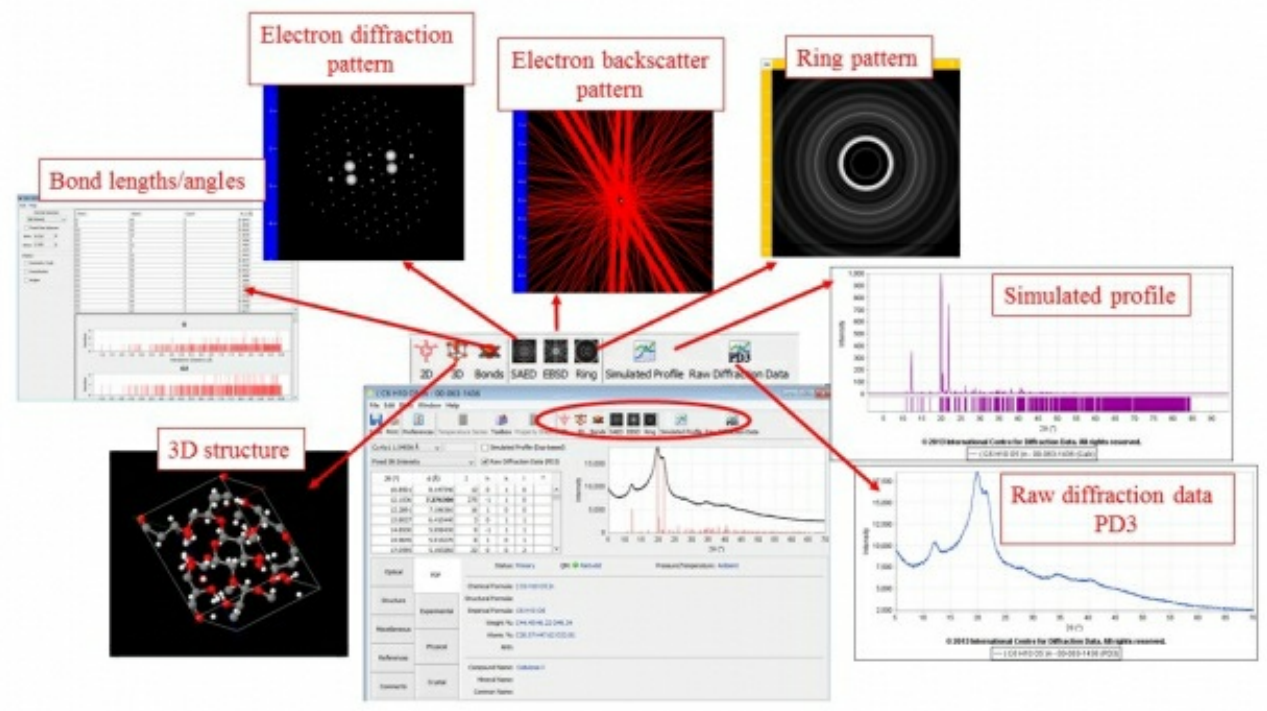

Keywords: polymer, powder XRD, phase identification 